\title{
SPANISH TERMS
}

A pulmón

Asociación de Vecinos

Blanco

Blanquear

Brigada de senderos

Brigadista

Buena onda

Cámara de Comercio

Charla

Comerciante

Comisión de Fomento

Compañeros de rubro

Comuna

Empresario

Escalador

Estanciero

Fantasma social

Gente humilde

Golondrina

Hostería

Kirchnerismo

Los Kirchners

Mirador

Negro

No deje rastro

Paisano

Porteño

Prestador

Recorrida

Refugio

Retenciones

Seccional
By lung, by sheer will, by the bootstraps

Association of Neighbors

White, formal, visible

Whiten, launder, make legitimate

Trail crew

Seasonal ranger

Good vibes

Chamber of Commerce

Informal talk

Small-business owner

Development Commission

(state-appointed administrative council)

Market-sector partners

Collective, community

Big-business owner

Climber

Ranch or farm owner

Social ghost

Humble or common folk

Patagonian swallow, seasonal

service worker

Bed-and-breakfast

Political ideology of the Kirchner-Fernández

governments

The Kirchners as individuals

Scenic viewpoint

Black, informal, obscured

Leave no trace (rules of outdoor conduct)

Gaucho, horseman, agrarian laborer

Person from Buenos Aires

Guiding company owner

Patrol

Chalet, hut

Export taxes

Park ranger station 
Tierra fiscal

Tirolesa

Villa
Public land (administered by the Consejo Agrario Provincial) Tyrolean traverse (a cable spanning a river or chasm) 